\title{
Luminosity optimization schemes in Compton experiments based on Fabry-Perot optical resonators
}

\author{
Alessandro Variola* and Fabian Zomer \\ Laboratoire de l'Accélérateur Linéaire (LAL), Université Paris-Sud Orsay, CNRS/IN2P3, 91898 Orsay, France \\ Eugene Bulyak, Peter Gladkikh, and Vladislav Skomorokhov \\ National Science Center, Kharkov Institute of Physics and Technology (NSC KIPT), Kharkov 61108, Ukraine \\ Tsunehiko Omori and Junji Urakawa \\ High Energy Research Organization (KEK), Oho 1-1, Tsukuba, Ibaraki 305-0801, Japan
}

(Received 17 May 2010; published 16 March 2011)

\begin{abstract}
The luminosity of Compton x-ray and $\gamma$ sources depends on the average current in electron bunches, the energy of the laser pulses, and the geometry of the particle bunch to laser pulse collisions. To obtain high power photon pulses, these can be stacked in a passive optical resonator (Fabry-Perot cavity) especially when a high average flux is required. But, in this case, owing to the presence of the optical cavity mirrors, the electron bunches have to collide at an angle with the laser pulses with a consequent luminosity decrease. In this article a crab-crossing scheme is proposed for Compton sources, based on a laser amplified in a Fabry-Perot resonator, to eliminate the luminosity losses given by the crossing angle, taking into account that in laser-electron collisions only the electron bunches can be tilted at the collision point. We report the analytical study on the crab-crossing scheme for Compton gamma sources. The analytical expression for the total yield of photons generated in Compton sources with the crab-crossing scheme of collision is derived. The optimal collision angle of the bunch was found to be equal to half of the collision angle. At this crabbing angle, the maximal yield of scattered off laser photons is attained thanks to the maximization, in the collision process, of the time spent by the laser pulse in the electron bunch. Estimations for some Compton source projects are presented. Furthermore, some schemes of the optical cavities configuration are analyzed and the luminosity calculated. As illustrated, the four-mirror two- or three-dimensional scheme is the most appropriate for Compton sources.
\end{abstract}

DOI: 10.1103/PhysRevSTAB.14.031001

PACS numbers: 29.27.Eg, 41.60.- $\mathrm{m}, 41.75 . \mathrm{Ht}$

\section{INTRODUCTION}

The high-energy physics community is carrying out a thorough research in the field of $\gamma$-ray production from laser-electron Compton scattering to explore the feasibility of intense polarized positron sources for the next lepton colliders like the ILC [1], the CLIC [2], and the SuperB [3]. These are the future pioneering accelerators of the post-LHC era and the related physics program is extremely ambitious. On the one hand, after exploiting the discovery potential of the LHC in the field of the Higgs physics and in the scenarios beyond the standard model (supersymmetry, terascale generation, extra dimensions, dark matter, superstrings, etc.), considerable progress will be made thanks to a lepton collider for precision measurements [4]. In all these projects the importance of providing a polarized positron source has been proven. In the ILC and CLIC there is a

\footnotetext{
*variola@lal.in2p3.fr

Published by American Physical Society under the terms of the Creative Commons Attribution 3.0 License. Further distribution of this work must maintain attribution to the author(s) and the published article's title, journal citation, and DOI.
}

strong physics case, whereas in the B factory the positron polarization would make it possible to increase the luminosity setting the machine parameters. Another exciting challenge will be to push the experimental range of the $e-p$ collider to the terascale as suggested in the LHeC project [5].

In this context the innovative scheme of polarized positron sources by Compton scattering is extremely ambitious since sources providing a flux of $\sim 10^{14}-10^{16} / \mathrm{s}$ circular polarized gamma are required. These very high-energy gamma rays are produced by collision of electron bunches at $\sim 1.0-2 \mathrm{GeV}$ (stored in an accumulation ring [1] or recirculated in an energy recovery linac (ERL) [6]) and laser pulses stored in optical cavities. They are subsequently converted in pairs of linear polarized electronspositrons in a tungsten target and collected in an adiabatic matching device. For example, to attain the required ILC gamma flux $\left(\sim 3 \times 10^{16} \mathrm{\gamma} / \mathrm{s}\right)$, it is necessary to collide with a high repetition frequency $(\sim 40 \mathrm{MHz})$ electron bunches of a few $n C$ with stored photon pulses of $\sim 0.1-0.5$ joule intensity. This source would be a pioneering $\gamma$ factory and could have an important impact on the possible construction of a $\gamma-\gamma$ collider [7] or for nuclear physics gamma factories [8]. 
In the low energy range, the interest in Compton scattering is exponentially increasing owing to the interest in highflux x-ray ultrafast pulses. Moreover, their production by means of a low energy accelerator ensures the device compactness that is particularly suitable for industrial, nuclear, and medical applications. Different devices exploiting this technique are already operating worldwide and other ambitious programs are under way [9-11]. The list of the different applied and industrial fields that are from this technique is long. In the medical field the possible applications are, among the others, in the field of static and dynamic imaging [12] (like 3D compressionless mammography [13] and bronchography), K-edge radiography and therapy, and catheterless coronary arteries angiography [14] also thanks to the use of the phase contrast method. The tunability at a specific wavelength provides new solutions also for gadolinium-based cancer therapy and blood imaging [15]. The importance of the monochromaticity and of the $K$-edge interaction in biological tissue and in the contrast products like iodine is extensively described in [13], where the imaging and therapy application are summarized. An applied physics field that benefits from compact Compton sources is X-ray diffraction protein crystallography $[9,15]$, where a performance close to the light sources one is attained. This performance provides also a range of chemistry analysis techniques applications, like, for example, $\mathrm{x}$-rays diffraction, $\mathrm{x}$-ray fluorescence, and $\mathrm{x}$-ray absorption near edge structure, for applications in the cultural heritage preservation domain [16]. The possibility of identifying the atomic number of different materials with hard $\mathrm{x}$ rays also opens the way for nuclear application of the Compton scattering $[17,18]$. High penetration photons are also suitable for security and aerospace applications; for example, the radiography of shielded material investigating the presence for small quantities of a particular nuclei [18].

In all these applications the requirements are different. Flux ranges between $10^{6}$ to $10^{13} \mathrm{ph} / \mathrm{s}$ with energy that varies from few $\mathrm{keV}$ in medical science to few $\mathrm{MeV}$ for nuclear applications. Pulse length constraints can be very demanding in ultrafast biochemistry studies (in the 100 fs range).

Therefore, depending on the energy of the impinging electron beam, the Compton associated technology will have a fundamental impact on both fundamental and applied research. This is due to the fact that Compton scattering is, by far, the physical effect that most efficiently boosts the photon energy. In the past, it has been used in very specific applications owing to the extremely low cross section but, at present, the impressive increase in lasers and electron source technologies allows one access to unexplored flux ranges for different possible applications in the above-mentioned fields. In this framework two different regimes can be identified: (a) the high instantaneous flux where an important number of photons are scattered in a short pulse at low repetition frequency; (b) the high average flux where a lower pulse energy is produced but with a very important collision repetition frequency $\left(f_{\text {rep }}\right)$.

In the latter case, the possibility of amplifying the laser pulse in a passive optical resonator (Fabry-Perot cavity) should make it possible, in the future, to operate Compton collisions on a stored pulse in the MW power regime at a very high $f_{\text {rep }} \sim 10 \mathrm{Mhz}-1 \mathrm{GHz}$. Passive resonators are made of extremely high reflectivity mirrors that need precise requirements in manufacturing technology and cavity cleanness. But the effective scattered photon flux can be increased not only by upgrading the technology in high repetition frequency lasers and in high current accelerator, but also by carefully optimizing the interaction in the collision point. This is particularly important in some cases (high finesse mirrors, high scattered flux) where a collision angle is required to preserve the optical resonator mirror coatings.

In this paper we have taken into account different aspects of luminosity optimization concerning both the electron bunches and the laser pulse. We will show how the choice of the appropriate electron bunch crab angle can almost completely suppress the strong flux reduction given by the collision angle when dealing with particle bunches much longer than laser pulses. For its peculiarity, this scheme should also be applied in particle colliders, like the LHeC project linac-ring scheme, where a short ERL electron bunch interacts with a long LHC hadron bunch [19]. We will also illustrate a way of optimizing the luminosity by obtaining extremely small laser waists with a four-mirrors mechanical stable optical cavity adjusting the Fabry-Perot resonator pulse mode to the electron beam shape.

\section{PROBLEM DEFINITION-LUMINOSITY AND YIELD}

The rate of high-energy photons scattered off by a bunch of relativistic electrons crossing a laser pulse can be presented as the result of "the power" $(P)$ and "the geometric" $(G)$ factor [20],

$$
R=\sigma L=\sigma P G,
$$

where $R$ is the rate, $L$ the luminosity, and $\sigma$ the cross section of the scattering process (Compton cross section).

The power factor is

$$
P=f_{\text {rep }} N_{\mathrm{e}} N_{\mathrm{ph}}=N_{\mathrm{ph}} I_{\mathrm{e}} / e,
$$

where $N_{\mathrm{e}}$ and $N_{\mathrm{ph}}$ are, respectively, the number of electrons in the bunch and photons in the laser pulse, $I_{\mathrm{e}}$ is the average current of the electron beam, and $f_{\text {rep }}$ is the frequency of bunches crossing through the interaction point (IP) which is equal to the number of bunch-to-pulse collisions per second.

The geometric factor, which represents the space-time overlap of the two bunches, can be presented as

$$
G(\phi)=(1+\beta \cos \phi) \int_{V} \int_{T} n_{\mathrm{e}}(V, t) n_{\mathrm{ph}}(V, t) d V d t,
$$


where $\phi$ is the crossing angle, $n_{\mathrm{e}}$ and $n_{\text {las }}$ the density distributions of electrons and photons normalized to unity, and $\beta=v_{e} / c$ is the ratio of the electron speed to the speed of light. The integration has to be performed over the volume $V$ and duration $T$ of a single crossing of the bunch with the laser pulse. Thus, to maximize the yield of the high-energy photons from the Compton source, one has to reasonably maximize both the power factor, i.e., the average current of the electron beam and the energy contained in the laser pulse, and the geometric factor. The optimization of the geometric factor is less constrained, since there are fewer physical and technological limitations, such as the space charge effects and the consumed power as far as the accelerators are concerned or the maximum attainable lasers power and power load on the optical resonator mirrors. The geometric factor, for tri-Gaussian bunches and pulses crossing along axes of the ellipsoids in the $x$, $y$ plane at an angle $\phi$, for the case of ultrarelativistic electrons, $1-\beta \ll 1$, reads (see [21])

$$
G(\phi)=\frac{1}{2 \pi \sqrt{\sigma_{z}^{2}+\sigma_{z}^{\prime 2}} \sqrt{\sigma_{x}^{2}+\sigma_{x}^{\prime 2}+\left(\sigma_{y}^{2}+\sigma_{y}^{\prime 2}\right) \tan ^{2} \frac{\phi}{2}}},
$$

where $\sigma_{x, y, z}$ are the rms dimensions of electron bunch; once primed they are the respective dimensions of the laser pulse. In the case of strong laser or beam focalization (electron bunch length shorter than the IP beta function and laser beam Rayleigh length shorter of the electron bunch length), the hourglass effect has to be taken into account by considering the transverse beams sizes as a function of the longitudinal coordinate $z$, i.e., $\sigma_{x}, \sigma_{y}, \sigma_{x^{\prime}}, \sigma_{y^{\prime}}=\sigma_{x}(z), \sigma_{y}(z), \sigma_{x^{\prime}}(z), \sigma_{y^{\prime}}(z)$. In the next paragraph the luminosity optimization is carried out without taking into account a longitudinal dependence of the beams sizes in the interaction region (hourglass effect). This allows to better understand the proposed scheme. The calculations considering the hourglass effect are illustrated in Appendix A.

As it can be seen from (4), the geometric factor is inversely proportional to the dimensions of both the bunch and the pulse and of the crossing angle $\phi$, which should be kept as small as possible to enhance the photon flux.

\section{CRAB CROSSING IN COMPTON SOURCES}

From (4) it follows that the contribution of the vertical dimensions normal to the plane of crossing $[z$ axis in the assumption used in Eq. (4)] is decoupled from that in the collision plane $x, y$. In practical cases, for a non-head-on collision, the dominant reduction in the geometric factor arises from the length of the electron bunches. It is well known that in storage rings the bunch length cannot be significantly reduced at the collision point as done by the focusing systems in the case of the transverse dimensions. To mitigate the effect of the bunch length, the crabcrossing scheme was proposed for the Compton sources
[22]. In the crab-crossing scheme only the electron bunch is turned by an angle with respect to velocity of electrons, see Fig. 1, to enhance the yield.

\section{Theory of crab crossing}

The main goal of this section is to determine an analytical expression for the total yield of photons generated in Compton backscattering of a laser pulse and an electron bunch in the case of a crab-crossing collision. For this purpose it will be assumed that the electron bunch and photon bunch (laser pulse) have an ellipsoid shape; a Gaussian distribution is assumed as far as the densities of electrons and photons are concerned; we consider the collisions in the Cartesian laboratory coordinate frame. A planar symmetry is initially assumed, that is the axes of the electron and photon bunches lie in the same plane. We make use of the procedure described in [20] and extend it to take into account the bunch ellipsoid axes tilt with respect to the electrons trajectory. Let us consider the crossing of the photon bunch (laser pulse) and the electron bunch in the laboratory coordinate frame $x, y, z$ (see. Fig. 1).

The electron bunch is propagating along the $y$ axis with velocity $v_{\mathrm{e}}$ in the laboratory frame $(x, y, z)$; the photon pulse contrapropagates along the $y^{\prime}$ axis in coordinate frame $\left(x^{\prime}, y^{\prime}, z^{\prime}\right)$ turned by an angle $\phi$ in respect to the $y$ axis.

The tri-Gaussian density distribution in both the bunch and the pulse is assumed. The distribution functions of electron and photon bunches in (3) read

$$
\begin{aligned}
n_{\mathrm{e}} & =\frac{1}{(2 \pi)^{3 / 2} \sigma_{x} \sigma_{y} \sigma_{z}} \exp \frac{1}{2}\left[-\frac{x^{\prime \prime 2}}{\sigma_{x}^{2}}-\frac{y^{\prime \prime 2}}{\sigma_{y}^{2}}-\frac{z^{\prime 2}}{\sigma_{z}^{2}}\right], \\
n_{\mathrm{ph}} & =\frac{1}{(2 \pi)^{3 / 2} \sigma_{x}^{\prime} \sigma_{y}^{\prime} \sigma_{z}^{\prime}} \exp \frac{1}{2}\left[-\frac{x^{\prime 2}}{\sigma_{x}^{\prime 2}}-\frac{\left(y^{\prime}+T\right)^{2}}{\sigma_{y}^{\prime 2}}-\frac{z^{\prime 2}}{\sigma_{z}^{\prime 2}}\right],
\end{aligned}
$$

where $T=c t$ is the time variable.

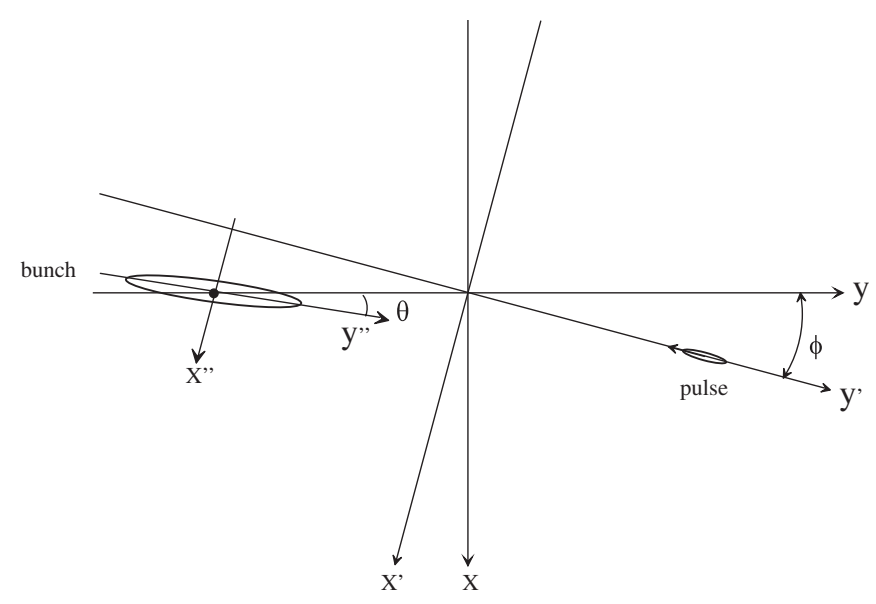

FIG. 1. The electron and photon coordinate frames; $z$ coincides with $z^{\prime}$ and directed upward from the $(x, y)$ plane. 
The electron coordinates in the bunch frame relate to the lab frame as

$$
\begin{aligned}
& x^{\prime \prime}=x \cos \theta+(y-\beta T) \sin \theta ; \\
& y^{\prime \prime}=-x \sin \theta+(y-\beta T) \cos \theta ; \\
& z^{\prime \prime}=z,
\end{aligned}
$$

and the photons frame to the lab's as

$$
\begin{gathered}
x^{\prime}=x \cos \phi+y \sin \phi ; \quad y^{\prime}=-x \sin \phi+y \cos \phi ; \\
z^{\prime}=z,
\end{gathered}
$$

with $\theta$ being the crabbing angle.

By substituting the explicit relations for the distribution functions (5) and collecting the like terms, the geometric factor (3) takes the form

$$
G=\frac{g}{(2 \pi)^{3}} \iiint \int \exp \left[-\frac{\mathrm{F}(x, y, z, T)}{2}\right] d x d y d z d T,
$$

where

$$
g=\frac{(1+\beta \cos \phi)}{\sigma_{x} \sigma_{y} \sigma_{z} \sigma_{x}^{\prime} \sigma_{y}^{\prime} \sigma_{z}^{\prime}},
$$

and

$$
\begin{aligned}
\mathrm{F}(x, y, z, T)= & a_{11} x^{2}+a_{22} y^{2}+a_{33} z^{2}+a_{44} T^{2}+2 a_{12} x y \\
& +2 a_{14} x T+2 a_{24} y T .
\end{aligned}
$$

The coefficients $a_{i j}$ in expression (9) are calculated as

$$
\begin{aligned}
& a_{11}=\frac{\cos ^{2} \theta}{\sigma_{x}^{2}}+\frac{\sin ^{2} \theta}{\sigma_{y}^{2}}+\frac{\cos ^{2} \phi}{\sigma_{x}^{\prime 2}}+\frac{\sin ^{2} \phi}{\sigma_{y}^{\prime 2}} ; \\
& a_{22}=\frac{\sin ^{2} \theta}{\sigma_{x}^{2}}+\frac{\cos ^{2} \theta}{\sigma_{y}^{2}}+\frac{\sin ^{2} \phi}{\sigma_{x}^{\prime 2}}+\frac{\cos ^{2} \phi}{\sigma_{y}^{\prime 2}} ; \\
& a_{12}=\left(\frac{1}{\sigma_{x}^{2}}-\frac{1}{\sigma_{y}^{2}}\right) \cos \theta \sin \theta+\left(\frac{1}{\sigma_{x}^{\prime 2}}-\frac{1}{\sigma_{y}^{\prime 2}}\right) \cos \phi \sin \phi ; \\
& a_{14}=-\beta\left(\frac{1}{\sigma_{x}^{2}}-\frac{1}{\sigma_{y}^{2}}\right) \cos \theta \sin \theta-\frac{\sin \phi}{\sigma_{y}^{\prime 2}} ; \\
& a_{24}=\frac{\cos \phi}{\sigma_{y}^{\prime 2}}-\frac{\beta \sin ^{2} \theta}{\sigma_{x}^{2}}-\frac{\beta \cos ^{2} \theta}{\sigma_{y}^{2}} ; \quad a_{33}=\frac{1}{\sigma_{z}^{2}}+\frac{1}{\sigma_{z}^{\prime 2}} .
\end{aligned}
$$

After reduction of the relation (9) to the diagonal form and integration of (8) over spatial and temporal infinite limits, we obtain

$$
G(\phi, \theta)=\frac{1}{2 \pi \sqrt{\sigma_{z}^{2}+\sigma_{z}^{\prime 2}} \sqrt{f_{\mathrm{e}}(\phi, \theta)+f_{\mathrm{ph}}(\phi)}},
$$

where $f_{\mathrm{e}}(\phi, \theta)$ and $f_{\mathrm{ph}}(\phi)$ are functions of the electron bunch and laser pulse dimensions, and of the crossing and crab angles:

$$
\begin{aligned}
f_{\mathrm{e}}(\phi, \theta)= & \sigma_{x}^{2}\left(\frac{\cos (\theta-\phi)+\beta \cos \theta}{1+\beta \cos \phi}\right)^{2} \\
& +\sigma_{y}^{2}\left(\frac{\sin (\theta-\phi)+\beta \sin \theta}{1+\beta \cos \phi}\right)^{2}, \\
f_{\mathrm{ph}}(\phi)= & \sigma_{x}^{\prime 2}+\beta^{2} \sigma_{y}^{\prime 2}\left(\frac{\sin \phi}{1+\beta \cos \phi}\right)^{2} .
\end{aligned}
$$

For the most interesting case of ultrarelativistic electrons, $\beta \approx 1$, the functions (11) take a simpler form

$$
\begin{aligned}
f_{\mathrm{e}}(\phi, \theta)= & \sigma_{x}^{2}\left(\frac{\cos (\theta-\phi)+\cos \theta}{1+\cos \phi}\right)^{2} \\
& +\sigma_{y}^{2}\left(\frac{\sin (\theta-\phi)+\sin \theta}{1+\cos \phi}\right)^{2}, \\
f_{\mathrm{ph}}(\phi)= & \sigma_{x}^{\prime 2}+\sigma_{y}^{\prime 2}\left(\frac{\sin \phi}{1+\cos \phi}\right)^{2},
\end{aligned}
$$

and the generalized geometric factor (10) is reduced to the particular case (4) for $\theta=0$ (no crabbing).

The maximal value of the geometric factor is attained (in the ultrarelativistic case) at the crabbing angle exactly equal to half of the crossing angle, as can be easily found from (12a):

$$
\max G(\phi, \theta) \rightarrow \min f_{\mathrm{e}}(\phi, \theta) ; \quad \theta=\phi / 2 .
$$

This condition eliminates the second term in (12a), so the yield of the scattered off photons becomes independent from the length of the electron bunch. Physically this means that the shorter laser pulse spends all the time inside the long electron bunch during the crossing, thanks to the crab angle optimization. It is for this reason that this scheme can be extended also to particle colliders where a short bunch interacts with a much longer one (like in a linac-ring interaction, see [19]). As it can be rigorously proved, the maximization condition, $\theta=\phi / 2$, is valid for any symmetric density distribution with transverse maximum along the longitudinal axis of the bunch: $\max n_{\mathrm{e}}\left(x^{\prime \prime}, y^{\prime \prime}, z^{\prime \prime}\right)=n_{\mathrm{e}}\left(0, y^{\prime \prime}, 0\right)$.

The geometric factor for optimal crabbing angle $G_{\max }(\phi) \equiv G(\phi, \theta=\phi / 2)$ reads

$$
G_{\max }(\phi)=\frac{1}{2 \pi \sqrt{\sigma_{z}^{2}+\sigma_{z}^{\prime 2}} \sqrt{\sigma_{x}^{2} \sec ^{2} \frac{\phi}{2}+\sigma_{x}^{\prime 2}+\sigma_{y}^{\prime 2} \tan ^{2} \frac{\phi}{2}}},
$$

and the yield enhancement from the crab crossing over the regular one (4),

$$
\frac{G_{\max }(\phi)}{G(\phi, 0)}=\sqrt{\frac{\left(\sigma_{x}^{2}+\sigma_{x}^{\prime 2}\right) \cos ^{2} \frac{\phi}{2}+\left(\sigma_{y}^{2}+\sigma_{y}^{\prime 2}\right) \sin ^{2} \frac{\phi}{2}}{\sigma_{x}^{2}+\sigma_{x}^{\prime 2} \cos ^{2} \frac{\phi}{2}+\sigma_{y}^{\prime 2} \sin ^{2} \frac{\phi}{2}}},
$$

increases with the laser pulse and electron bunch transverse dimensions decreasing. Moreover, for a very short laser 

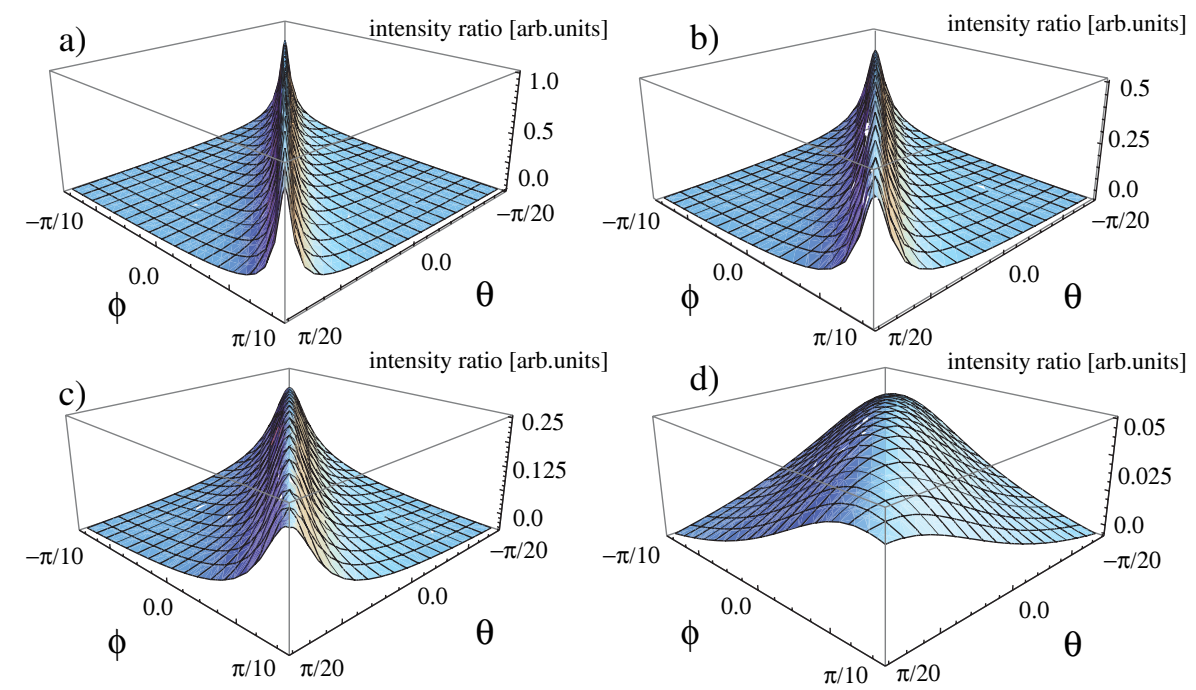

FIG. 2. Geometrical factor variation as a function of the crossing and the crab angles and of the electron bunch length.

pulse, $\sigma_{y}^{\prime 2} \tan ^{2} \frac{\phi}{2} \ll \sigma_{x}^{2}$, the yield approaches the most effective head-on one, $\phi=0$.

The parametric dependence of the proposed crabcrossing scheme is illustrated in Fig. 2. Here we simulated the collision between one electron bunch and one laser pulse and evaluated the geometrical factor as a function of the crossing $(\phi)$ and of the crabbing angle $(\theta)$ for four different electron bunch lengths. The laser pulse and the electron beam were always supposed to have the same transversal sizes $\sigma_{T}=50 \mu \mathrm{m}$. The laser pulse sigma length is supposed to be constant and equal to $300 \mu \mathrm{m}$ (1 ps). In the four different plots [(a), (b), (c), (d)] the electron bunch length was, respectively, varied at: (a) $=1.2 \mathrm{~cm}$, (b) $=6 \mathrm{~mm}$, (c) $=3 \mathrm{~mm}$, (d) $=0.6 \mathrm{~mm}$. The angular range of the plots is fixed as the crossing angle is twice the crabbing angle: $(-\pi / 10<\phi<$ $\pi / 10 ;-\pi / 20<\theta<\pi / 20)$. The intensity is normalized to the head-on case with a bunch length of $1.2 \mathrm{~cm}$, so the effectiveness of the scheme can be evaluated in the four cases. It is possible to appreciate that in all four cases the maximum yield condition at $\phi=2 \theta$ is satisfied. From the plots it is also evident how the scheme is much more efficient for a much more significant electron bunch length [case (a)]: in this case the maximization curve is very sharp and the condition on the crab angle must be strictly satisfied to obtain a significant intensity gain. The opposite is also evident in shortening the electron bunch, where the curve is broader. This means that for shorter bunch length the crab scheme does not introduce an important gain in respect to the head-on case. The curve sharpness defines, depending on the parameters, the precision required on the crab angle to obtain an effective flux enhancement. For a very long electron bunch the precision on the crab angle is fundamental. In the upper case for an electron bunch of $6 \mathrm{~mm}$ a crab angle error of $10 \mathrm{mrad}$ leads to a flux loss greater than a factor two.

\section{NUMERICAL RESULTS. SIMULATIONS}

Some numerical estimations were made to validate the above analytical results taking into account different applications on ongoing projects.

\section{A. ILC case-Flux losses due to the crossing angle and crab angle efficiency}

First of all, an estimation of the luminosity losses, due to the collision angle coupled with the electron bunch length, and of the effectiveness of the crab angle scheme is provided taking into account the ILC Compton ring based positron sources. To scan the two parameters (crossing angle and bunch length) we simulated the Compton production with the Monte Carlo code CAIN, considering a typical parameter set: (i) electron bunch: $\sigma_{x}=30 \mu \mathrm{m}$, $\sigma_{y}=5 \mu \mathrm{m}, \varepsilon_{x}=4.5 \mathrm{~nm}, \varepsilon_{y}=25 \mathrm{pm}, \beta_{y}=1 \mathrm{~m}, \beta_{x}=$ $20 \mathrm{~cm}$; (ii) photon pulse (laser): $\lambda=1.06 \mu \mathrm{m}$, waist size $\omega_{0}=20 \mu \mathrm{m}$, Rayleigh length $z_{R}=1.18 \mathrm{~mm}, \sigma_{z}=$ $0.3 \mathrm{~mm}$ (1 ps).

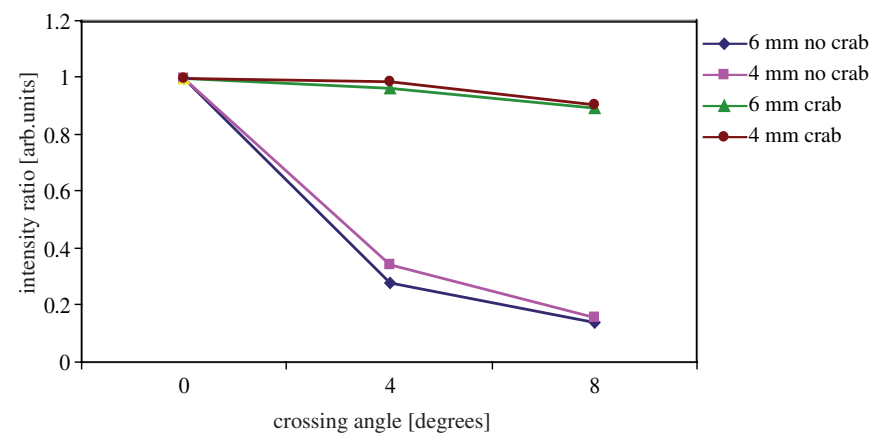

FIG. 3. ILC ring gamma flux for different collision angles. The effect of the crab-crossing scheme is remarkable, since the produced flux became quite insensitive to the crossing angle parameter. 


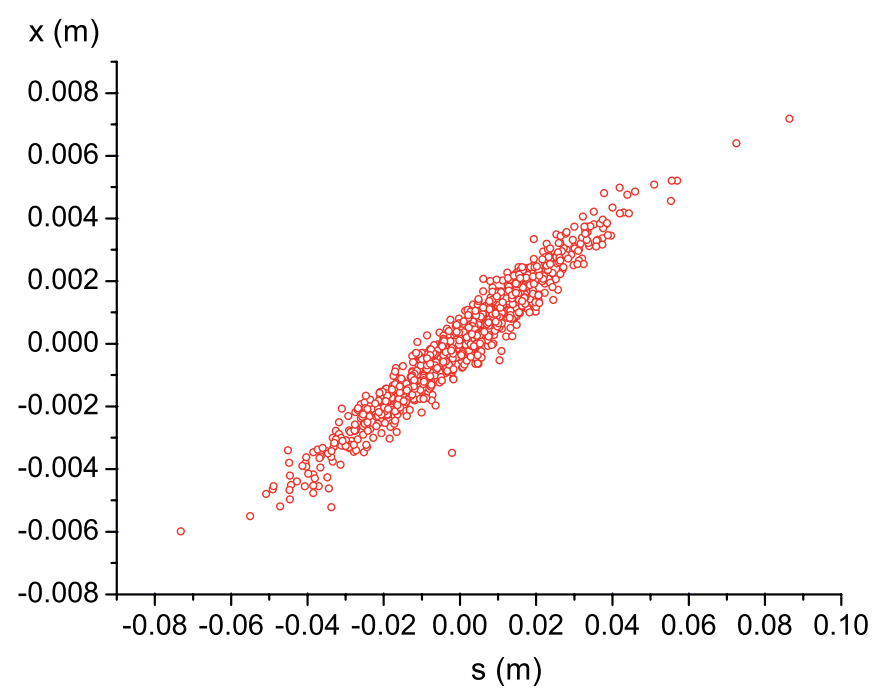

FIG. 4. Crabbed distribution of particles in the horizontal plane at the IP. It is possible to remark the coupling between the transverse $(x)$ and longitudinal $(s)$ coordinate in the collision point.

Crossing angles were varied from 0 (head-on case) to 8 degrees and two different bunch lengths were considered, respectively $\sigma_{z}=6 \mathrm{~mm}$ and $4 \mathrm{~mm}$. The results are illustrated in Fig. 3. First we have to point out that in this case the laser Rayleigh length is shorter than the electron bunch. This has been taken into account in the Monte Carlo simulations that demonstrated that, also in this case, the proposed method is effective. It is, in fact, possible to highlight that quite independently of the bunch length the missing overlap due to the crossing angle is strongly suppressing the rate of a factor $\sim 5$ for eight degrees crossing. It is also evident how the crab-crossing scheme restores the lost flux. For the eight degrees crossing the flux losses in respect to the head-on case are of the order of $10 \%$.

\section{B. Compton ring simulation}

Crab crossing is especially advantageous for x-ray and gamma sources based on the storage rings due to large electron bunch lengthening induced by Compton scattering. The energy spread in the bunch may reach a few percents [23]. At a moderate momentum compaction factor the electron bunch length is considerably larger than the length of the laser pulse, which drastically decreases the gamma beam intensity.

A simulation for the case of a Compton ring dedicated for the nuclear resonance fluorescent analysis [24] was worked out.

The main ring parameters are the following: (i) ring circumference $C=41.5 \mathrm{~m}$; (ii) electron energy $240-530 \mathrm{MeV}$; (iii) laser photons energy $E_{\mathrm{ph}}=$ $1.164 \mathrm{eV}$; (iv) laser flash energy stored in optical cavity $W_{\text {las }}=5 \mathrm{~mJ}$; (v) collision angle 12 degrees.

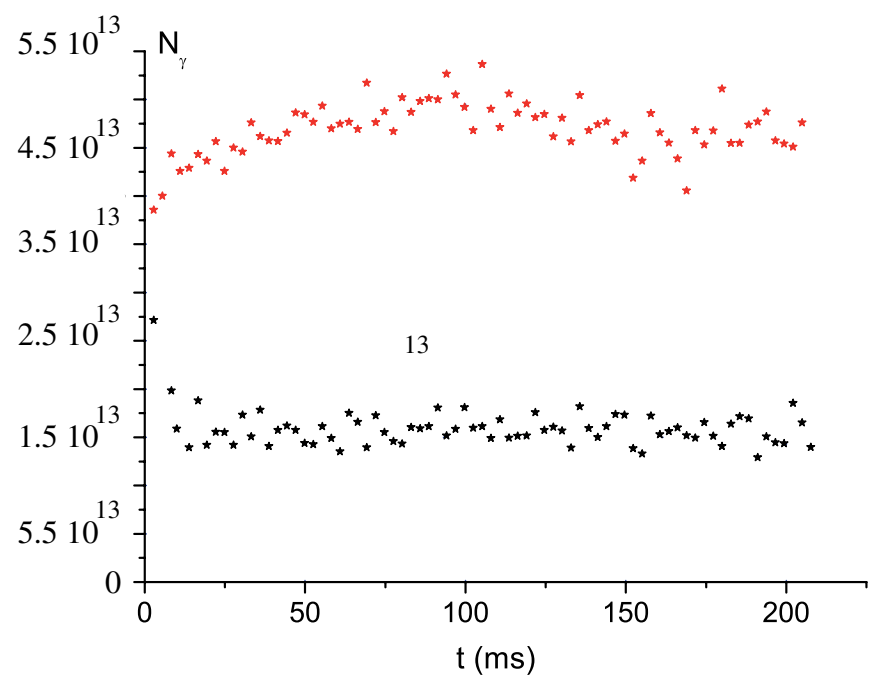

FIG. 5. Gamma beam intensity at crab crossing (red) and normal crossing (black) vs time.

The required crabbing angle, 6 degrees, is provided by two normal-conductive crab cavities with rf voltage $0.5 \mathrm{MV}$.

The effect of the crab cavity is simulated taking into account the transverse kick as a function of the longitudinal coordinate $s$ :

$$
\Delta x^{\prime}=\frac{e V_{C}}{E_{e}(1+\delta)} \sin \left(\frac{2 \pi}{\lambda} s\right)
$$

where $E_{\mathrm{e}}$ is the electron energy, $\delta$ deviation of the energy from the synchronous one, $\lambda \mathrm{rf}$ wavelength, and $s$ the longitudinal coordinate of a particle with respect to the synchronous one.

A simulated distribution of electrons over the phase space at the azimuth of IP is presented in Fig. 4, the gamma beam intensity at both normal and crab-crossing modes is presented in Fig. 5.

As one can see from Fig. 5, gamma beam intensity at normal crossing is quickly reduced because of electron bunch lengthening, while intensity at crab crossing is practically constant in time (during the working cycle). The steady state gamma beam intensity at crab crossing is approximately 3 times larger than the intensity at normal crossing. The simulations agree well with the analytical expression (4).

\section{Estimations for KEK $5 \mathrm{MeV}$ Compton experiment-Crab scheme efficiency}

Another experimental program is taking into account low energy Compton collisions for $\mathrm{x}$-ray production using a linac [25]. In this case the electron bunch and the laser pulse lengths are of the same order, but the collision geometry imposes an important crossing angle. The collision parameters for this proposal are listed in Table I. 
TABLE I. Parameters of the proposed $5 \mathrm{MeV}$ Compton experiment.

\begin{tabular}{lcc}
\hline \hline Parameter & Unit & Value \\
\hline Energy of electrons & $\mathrm{MeV}$ & 5 \\
Energy of laser photons & $\mathrm{eV}$ & 1.164 \\
Crossing angle & Degrees & 20 \\
Bunch at IP & & \\
Length $\left(\sigma_{y}\right)$ & $\mathrm{mm}$ & 1.2891 \\
Width $\left(\sigma_{x}\right)$ & $\mathrm{mm}$ & 0.1 \\
Height $\left(\sigma_{z}\right)$ & $\mathrm{mm}$ & 0.1 \\
Pulse at IP & & \\
Length $\left(\sigma_{y}^{\prime}\right)$ & $\mathrm{mm}$ & 1.2891 \\
Width $\left(\sigma_{x}^{\prime}\right)$ & $\mu \mathrm{m}$ & 10 \\
Height $\left(\sigma_{z}^{\prime}\right)$ & $\mu \mathrm{m}$ & 10 \\
\hline \hline
\end{tabular}

Also for this case we calculated the total Compton flux, respectively, for the nominal and the crab cases. First estimations are as follow: (i) maximal energy in Compton spectrum $E_{\mathrm{x}}^{\max }=371.42 \mathrm{eV}$; (ii) yield enhancement from the crab crossing 1.35 (by $35 \%$ ).

For the efficiency of the crab scheme the most critical parameter limiting the yield is the laser pulse length. In Fig. 6 the dependencies of yield on the laser pulse length are presented for both the normal and the crab collision (the purple vertical line indicates the initially provided parameters). It is again possible to appreciate how the crab scheme increases its efficiency when the laser pulse length is much lower than the electron beam one.

\section{OPTICAL CAVITIES FOR COMPTON SOURCES}

In this section the choice of the optimal laser system and Fabry-Perot cavity configuration will be considered. Besides parameters as the minimal dimensions of the laser pulse at the interaction point and the high pulse power, the crossing angle $\phi$ is also analyzed since it is mainly dependent on type and design of the optical cavity.

\section{A. Fabry-Perot cavity-Advantages and related problems}

Fabry-Perot resonators are passive optical cavities where a laser pulse is continuously stacked to enhance the circulating pulse energy [26]. The advantage of a passive resonator is given by the reduced required power of the laser source and by the power losses that are limited to the mirrors ones, owing to the absence of an active element inside the cavities. An extremely important average energy can be stored up to the limit imposed by the high reflectivity coatings mirrors technology. Optical resonators can be used in pulsed [27] or cw mode. The pulse mode makes it possible to store more energy in the single pulse but requires an important development in the lasercavity locking, especially at very high gain owing to the extremely sharp cavity passband. For Compton experiments the use of Fabry-Perot resonators implies some

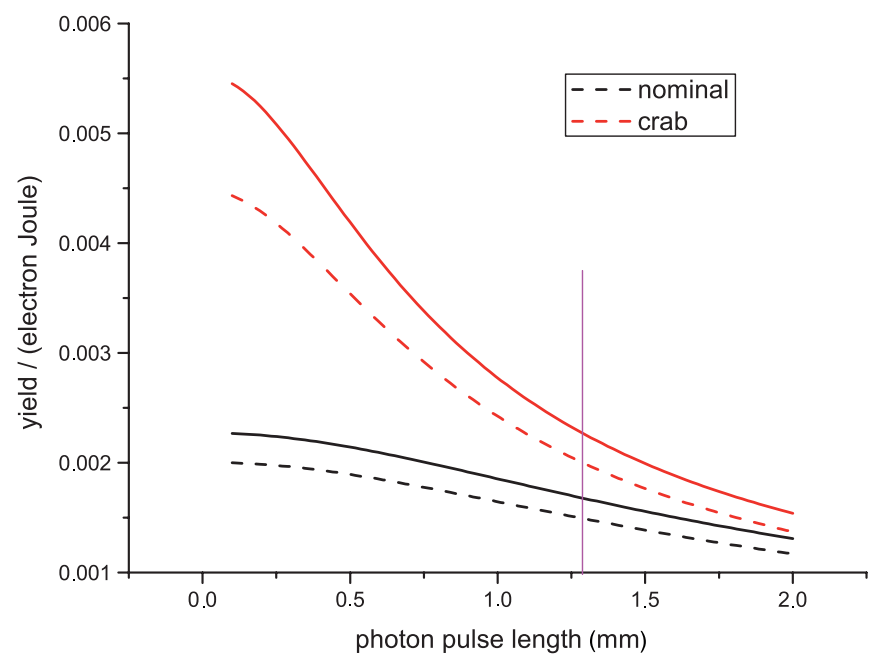

FIG. 6. Yield vs laser pulse length. Solid curves for the laser pulse $\sigma_{x, z}^{\prime}=10 \mu \mathrm{m}$, dashed $\sigma_{x, z}^{\prime}=50 \mu \mathrm{m}$.

evident advantages but also some constraints that can affect the system efficiency.

The main advantages are: (i) power enhancement factor. At present an enhancement of more than a factor $10^{3}$ has been obtained in the pulsed regime for pulses of $\sim 1$ ps and 6000 for longer pulses ( $\sim 30 \mathrm{ps})$ [28]. The $\mathrm{cw}$ cavities are limited by the mirror coating properties and can, in principle, enhance up to a factor $10^{6}$ [29]. (ii) The enhancement is not obtained by increasing the power of the laser or by adding active elements to the cavity. This strongly reduced the constraints on the laser average power (and consequently on its cost and dimensions). (iii) In a Fabry-Perot cavity, it is possible to enhance the power of a high repetition frequency laser. Therefore high $f_{\text {rep }}$ Compton collisions are allowed to increase the average flux. (iv) Fabry-Perot resonators have already been successfully used in cw regime for beam polarization measurements $[30,31]$ and in pulsed regime to produce gamma [32] and $x$ rays [33] in noisy accelerator environment showing good reliability. (v) The new technology of high power fiber laser seems to be applicable to optical resonators opening the way for the MW stored regime [34].

The main disadvantages of this scheme are related to the geometry and the stability of the Fabry-Perot resonators and their integration in the accelerator vacuum. It is also necessary to point out that the great advantage of these devices is the possibility to operate collisions at very high repetition frequency producing a consequent high average flux. Therefore only accelerators that are in $\mathrm{cw}$, or semi-cw pulsed regime, like storage rings or ERLs, are suitable to host optical cavities for Compton collisions.

The disadvantages can be summarized as follows: (i) The length of the cavity is inversely proportional to the collision frequency, therefore it must be matched to the accelerator interbunch length. (ii) High reflectivity is obtained by a high technology multilayer $\mathrm{SiO}_{2} / \mathrm{Ta}_{2} \mathrm{O}_{5}$ 
coating of silica substrates that does not withstand strong irradiation by the emitted Compton $x-\gamma$ rays. Since the Compton flux follows the electron bunch direction, for very high rate the head-on collision is difficult to integrate and a crossing angle, in the interaction point, must be taken into account. (iii) To obtain very small waist in the interaction region, the classical two spherical-mirror FabryPerot cavity must be operated in the nearly concentric mode [35] (the cavity length is $\sim$ twice the curvature radius of the mirrors). This is an extremely instable mode owing to the important displacements of the optical axis corresponding to very little displacement on the mirrors. To avoid this problem the cavity is usually locked in the confocal mode (cavity length $\sim$ to the radius of curvature) where the obtainable waists are of the order of $\sim 400-500 \mu \mathrm{m}$. (iv) To reduce the waist size, not affecting the cavity stability, different designs of four-mirrors folded cavities are possible. On the other hand, the need for a closed geometrical loop implies other geometrical constraints in the design phase and in the cavity integration. For four-mirror cavities the laser polarization effects must be taken into account [36]. (v) Usually a four-mirrors cavity must be compact and avoid having accelerator components (as quadrupoles) inside. It is possible to integrate in the cavity mirrors with holes to allow head-on collisions, but this will reduce the cavity gain.

For all these reasons the four-mirror cavities are designed for collision schemes envisaging a crossing angle $\phi$ (a reasonable range that allows the cavity components integration is between 2 and 12 degrees)

\section{B. Four-mirror Fabry-Perot resonators}

\section{The laser pulse optimization at the interaction point}

Apart from the electron beam manipulations another way to optimize the geometrical luminosity factor, and so to maximize the luminosity, is to shape the laser beam at the IP. As previously said in a passive optical resonator it is not possible to insert gratings or other optical elements to shape the circulating pulse. So the only way to maximize the geometrical factor is to match the cavity eigenmodes to the collision geometry. A basic example is given by Compton rings where vertical emittance is much lower than the horizontal one. In this case in fact the electron bunch shape in the IP is an ellipse, but this is not the case with a standard two-mirror optical resonator which provides a round waist in the fundamental mode. In two-mirror cavities a matched elliptical pulse can be obtained by modes associated with elliptical mirrors. Nevertheless it is important to stress that, if the goal is a very high cavity gain, high reflectivity mirrors with geometries different from the spherical and the planar one can be very difficult and very expensive to manufacture. At present, to our knowledge, there are no experimental validations of elliptical mirrors at high reflectivity.

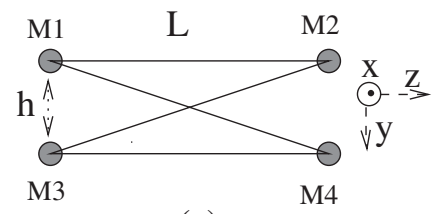

(a)

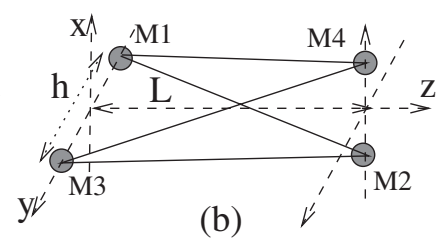

(b)
FIG. 7. Schematic views of four-mirror 2D bow-tie (a) and 3D tetrahedron (b) Fabry-Perot cavities. The mirrors $M 1$ and $M 2$ are flat and the mirrors $M 3$ and $M 4$ are spherical.

In the optical resonator introduction, it was stressed that producing very little waists and preserving the cavity mechanical stability is possible by designing a four-mirror resonator. This can be defined as a $2 \mathrm{D}$ resonator if all the four-mirror centers are lying in the same plane, and 3D if this condition is not satisfied (see Fig. 7).

In [36], we have shown that 2D and 3D four-mirror cavities are equally stable when the dimensionless parameter $h / 2 L<0.1$ and that $3 \mathrm{D}$ cavities are stable for any value of $h / 2 L$ (see Fig. 7 for the definitions of $h$ and $L)$. In addition, it was also shown in [36], and demonstrated experimentally in [37], that the eigenmode of the tetrahedron 3D cavity is circularly polarized. The 3D configuration therefore offers circularly polarized eigenmodes and a high level of stability with respect to the 2D geometry. However, the eigenmode of the 3D cavity belongs to the class of general astigmatic beams [38]; that is, the beam intensity profile is elliptical and the ellipse's eigen axes are turning during the propagation. Such a beam property will influence the Compton luminosity and the integration design of the cavity itself. It is evident in fact that, both for vacuum and wakefield requirements, the pulse introduction slit in the accelerator vacuum chamber must be minimized and its shape optimized. The possibility to introduce an ellipsoidal pulse in one direction and to rotate it in the IP adds an important degree of freedom in this system design, and give the possibility to match the laser pulse to the electron beam in such a way to maximize the geometrical factor and so the luminosity.

\section{2D-3D cavities example}

The impact of the cavity four-mirrors configuration on the luminosity strongly depends on the specific accelerator and laser beam parameters. Here we will provide some examples of numerical integration results for the $2 \mathrm{D}$ and 3D geometries. As far as the electron bunch is concerned, we take into account the parameters concerning a future experiment foreseen in Accelerator Test Facility (ATF)KEK where the collision point is located in a section characterized by very smooth $\beta_{s}$ variations. A numerical MATLAB code was developed to compute the propagation of the more complex 3D fundamental mode (the formalism of [38] was used and further compared to the more recent and compact formalism of [39]). The same code was also 
used to calculate the eigenmode of the 2D geometry subsequently validated by a much faster and simpler code based on the $A B C D$ matrix formalism (which holds for the 2D paraxial case). Once the eigenmode has been determined inside the cavity for a given geometrical configuration, the general luminosity formula [see Eqs. (1)-(3)] is used to numerically compute the luminosity. As far as the 3D case is concerned, the cavity is represented in the simplified scheme illustrated in Fig. 7: the radius of curvature of the spherical mirrors $M 3$ and $M 4$ are fixed to $R=500 \mathrm{~mm}$ so that the waist of the beam between these mirrors is controlled by the distance between $M 3$ and $M 4$. The total length of the cavity is finally controlled by the distance between the two flat mirrors $M 1$ and $M 2$. In order to match the second harmonic of the ATF bunch spacing, $c / 178.5 \mathrm{MHz}$, we obtain a total cavity round-trip length of $\sim 1.6 \mathrm{~m}$. The following numerical values for the last geometrical parameters are chosen: $h=100 \mathrm{~mm}$, $L=R+3.9 \mathrm{~mm}$, and electron bunch-laser beam crossing angle of $8^{\circ}$. As for the laser beam wavelength, we choose $=1060 \mathrm{~nm}$ as in Sec. IVA and the following electron beam parameters are considered: $\beta_{x}=25 \mathrm{~m}, \beta_{y}=6.3 \mathrm{~m}$, $\epsilon_{x}=10^{-9} \mathrm{~m}^{-1}, \epsilon_{y}=10^{-11} \mathrm{~m}^{-1}$, and $\sigma_{z}=9 \mathrm{~mm}$. The elliptical laser beam waists at the IP are, respectively, 45 and $93 \mu \mathrm{m}$. With these numbers, we obtain the laser beam intensity profile in the vicinity of the interaction point (i.e. halfway between the two spherical mirrors) shown in Fig. 8. Note that the coordinate system $(x, y, z)$ is related to the laser beam propagation axis so that $x$ and $y$ are transverse to this axis.

In order to compare the luminosity between the 3D and 2D cavity geometry, a 2D cavity is considered with
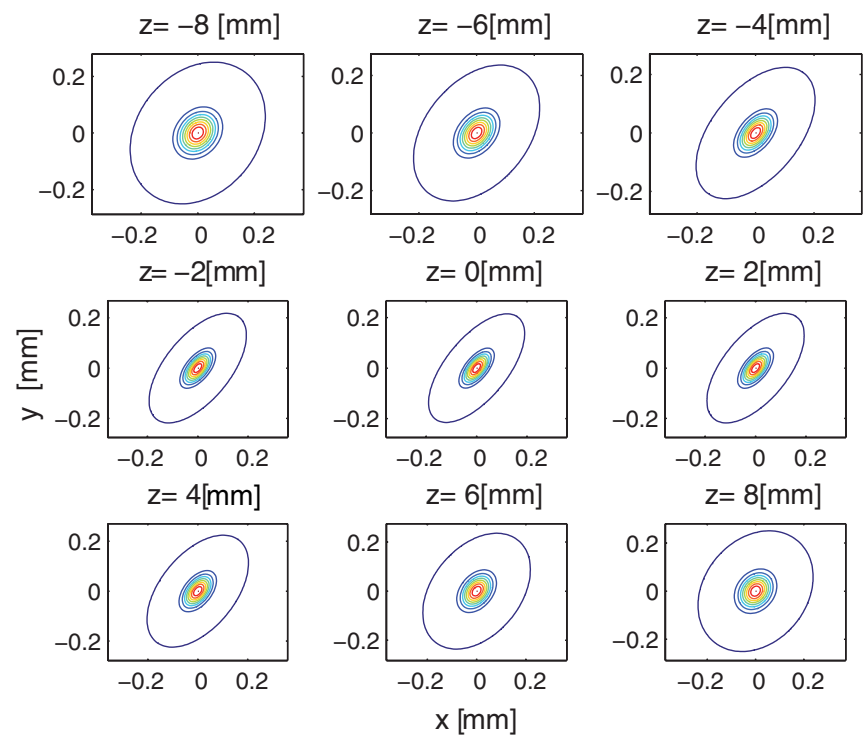

FIG. 8. Intensity profile of the 3D cavity eigenmode in a different plane closed to the laser beam waist $(z=0)$. The laser beam waists are 43 and $95 \mu \mathrm{m}$. The coordinate system $(x, y, z)$ is defined so that the axis $z$ is along the cavity optical axis.

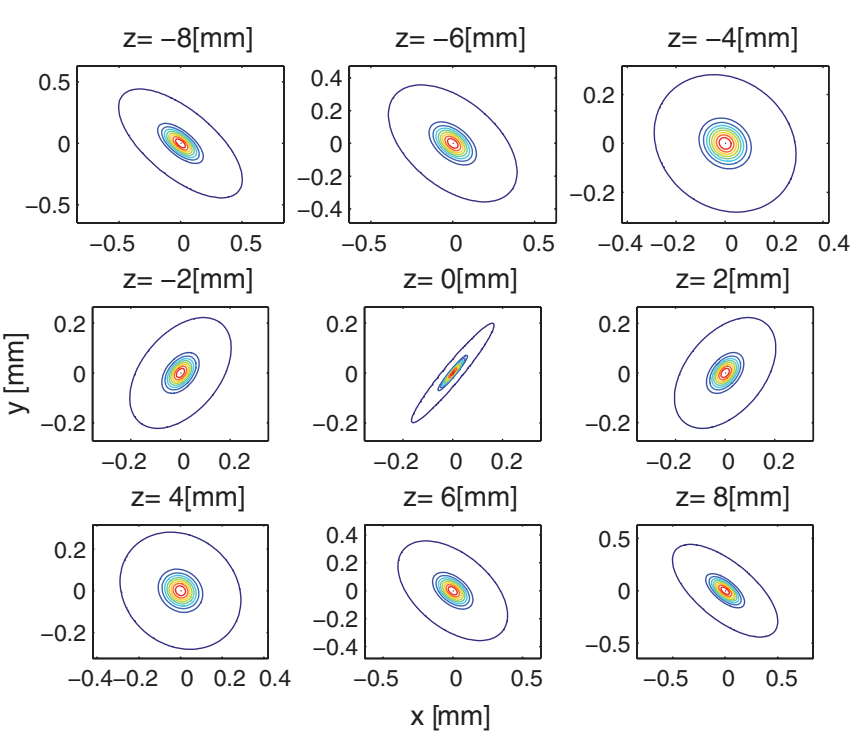

FIG. 9. Intensity profile of the 3D cavity eigenmode in a different plane closed to the laser beam waist $(z=0)$. The laser beam waists are 12 and $91 \mu \mathrm{m}$. The coordinate system $(x, y, z)$ is defined such that the axis $z$ is along the cavity optical axis.

the above-mentioned parameters $h=100 \mathrm{~mm}$ and $R=$ $500 \mathrm{~mm}$ but for a distance $L$ such that similar laser beam waists are obtained. For these specific parameters, the luminosity is found to be the same for the 2D and 3D geometries, essentially because of the $8^{\circ}$ crossing angle and of the relatively large electron bunch length of $12 \mathrm{~mm}$. As shown in [36], when the eigenmode starts to be divergent, the intensity profile is rotated by an angle $\sim 180^{\circ}$ between the two spherical mirrors and the smaller the laser beam waist (the higher the beam divergence), the shortest the distance over which this rotation takes place. This is illustrated in Fig. 9 where $L=R+3.53 \mathrm{~mm}$ was set leading to laser beam waists of 12 and $92 \mu \mathrm{m}$. Comparing to Fig. 8, one actually sees that the distance over which the axes of the elliptical intensity profile stays constant is reduced. This specific feature of general astigmatism introduces a correlation between size of the smaller laser beam waist and the electron bunch length: when reducing the laser beam waist one must, for a given crossing angle, reduce the electron bunch length in order to optimize the luminosity. This property of general astigmatic beams has to be taken into account when designing the IP region and especially the beam pipe aperture.

\section{SUMMARY AND DISCUSSION}

At present, Compton scattering-based machines have recently become rather popular for various applications. High-energy fundamental physics and applied medical and material science can profit from the fast technological advances that should provide emitted fluxes of order of 
magnitude higher than the present ones. In Compton schemes employing passive resonator cavities, when dealing with extremely high fluxes, careful optimization of the collision dynamics could lead to a significant increase in the emitted number of photons. We suggest two main ideas and the relative analytic treatment to calculate the efficiency of these schemes. The first consists in crabbing the charged particle beam to maximize the time effectively spent by the laser pulse inside the electron bunch. We provided the estimation for the gain in dependence of the angle of collision and for different bunch lengths. We highlighted that in the case of long electron bunches and short laser pulses the efficiency of this scheme, as far as the flux is concerned, can be very significant. The second proposal takes into account the transverse shape and rotation of the laser pulse in the interaction point varying the parameters of the 3D optical cavity. This takes into account the utilization of four-mirror cavities which can also provide very little waists maintaining the mechanical stability of the resonator. Experimental results on the mode excited in such a resonator will be illustrated in a future article. The use of a four-mirrors cavity is envisaged to produce an important gamma flux in the framework of a future experiment on ATF-KEK.

\section{ACKNOWLEDGMENTS}

The authors are grateful to the members of PosiPol collaboration for their valuable comments and helpful discussions.

\section{APPENDIX A: HOURGLASS EFFECT}

Accounting for the hourglass effect, the geometric factor in the luminosity (1), for a general case of an ellipsoidal electron bunch interacting with the Gaussian photon beam, reads

$$
G=\frac{(1+\cos \phi) R_{x} R_{z}}{(2 \pi)^{3 / 2} \sigma_{y} a_{1} a_{2}} \int_{-\infty}^{\infty} \frac{\exp \left(-\frac{u^{2}}{2 \sigma_{u}^{2}}\right) d u}{\sqrt{\left(\frac{R_{z}^{2}}{\sigma_{y}^{2}}+b_{1}^{2} u^{2}\right)\left(\frac{R_{x}^{2}}{\sigma_{y}^{2}}+b_{2}^{2} u^{2}\right)}},
$$

where $u=y^{\prime} / \sigma_{y}$ is a dimensionless variable; $\phi$ is the crossing angle; $\theta$ is the crabbing angle; $\sigma_{x}, \sigma_{y}$, and $\sigma_{z}$ are the dimensions of electron bunch; $\sigma_{y}^{\prime}$ is the length of photon pulse; $\sigma_{x 0}^{\prime}$ and $\sigma_{z 0}^{\prime}$ are the transversal dimensions of laser pulse at the waist point; and $R_{x, z}=\pi \sigma_{x 0, z 0}^{\prime 2} / \lambda$ is the Rayleigh parameter for each of transversal dimensions of photon pulse ( $\lambda$ is the laser wave length).

The coefficients in (A1) are

$$
\begin{aligned}
& a_{1}^{2}=\sigma_{z}^{2}+\sigma_{z 0}^{\prime 2} ; \quad b_{1}^{2}=\frac{\sigma_{z 0}^{\prime 2}}{\sigma_{z}^{2}+\sigma_{z 0}^{\prime 2}} \\
& a_{2}^{2}=\sigma_{x}^{2} \sigma_{y}^{2}+\sigma_{y}^{\prime 2}\left(\sigma_{x}^{2} \cos ^{2} \theta+\sigma_{y}^{2} \sin ^{2} \theta\right)+\sigma_{x 0}^{\prime 2}\left(\sigma_{y}^{\prime 2} \cos ^{2} \phi+\sigma_{x}^{2} \sin ^{2}(\phi-\theta)+\sigma_{y}^{2} \cos ^{2}(\phi-\theta)\right) ; \\
& b_{2}^{2}=\frac{\sigma_{x 0}^{\prime 2}\left(\sigma_{y}^{\prime 2} \cos ^{2} \phi+\sigma_{x}^{2} \sin ^{2}(\phi-\theta)+\sigma_{y}^{2} \cos ^{2}(\phi-\theta)\right)}{a_{2}^{2}}, \\
& a_{3}^{2}=\sigma_{x}^{2}[\cos (\phi-\theta)+\cos \theta]^{2}+\sigma_{y}^{2}[\sin (\phi-\theta)-\sin \theta]^{2}+\sigma_{y}^{\prime 2} \sin ^{2} \phi+\sigma_{x 0}^{\prime 2}(1+\cos \phi)^{2} ; \\
& b_{3}^{2}=\frac{\sigma_{x 0}^{\prime 2}(1+\cos \phi)^{2}}{a_{3}^{2}}, \quad \sigma_{u}^{2}=\frac{a_{2}^{2}}{\sigma_{y}^{2} a_{3}^{2}} \frac{\frac{R_{x}^{2}}{\sigma_{y}^{2}}+b_{2}^{2} u^{2}}{\sigma_{y}^{2}}+b_{3}^{2} u^{2}
\end{aligned}
$$

For a specific case of a round bunch, $\sigma_{x}=\sigma_{z}$, and the head-on collision, $\phi=\theta=0$, with a round laser pulse $\left(\sigma_{x 0}^{\prime}=\sigma_{z 0}^{\prime}\right)$, the geometric factor reduces to the known expression [40,41]:

$$
\begin{aligned}
\mathrm{G} & =\frac{2}{(2 \pi)^{3 / 2} a_{1} a_{1}} \int_{-\infty}^{\infty} \frac{\exp \left(-\alpha y^{\prime 2}\right) d y^{\prime}}{1+b^{2} \frac{y^{\prime 2}}{R^{2}}} \\
& =\frac{1}{\sqrt{2 \pi}} \frac{R}{b a_{1} a_{2}} \exp \left(\frac{\alpha R^{2}}{b^{2}}\right)\left[1-\operatorname{erf}\left(\frac{\sqrt{\alpha} R}{b}\right)\right],
\end{aligned}
$$

where

$$
\begin{array}{ll}
a_{1}^{2}=\sigma_{x}^{2}+\sigma_{x 0}^{\prime 2} ; & a_{1}^{2}=\left(\sigma_{x}^{2}+\sigma_{x 0}^{\prime 2}\right)\left(\sigma_{y}^{2}+\sigma_{y}^{\prime 2}\right) ; \\
b^{2}=\frac{\sigma_{x 0}^{\prime 2}}{\sigma_{x}^{2}+\sigma_{x 0}^{\prime 2}} ; & \alpha=\frac{2}{\sigma_{y}^{2}+\sigma_{y}^{\prime 2}} .
\end{array}
$$

If the electron bunch is much shorter than the Rayleigh parameter, $\sqrt{R_{z, x}^{2} / \sigma_{y}^{2}+b_{2}^{2} u^{2}} \approx R_{z, x} / \sigma_{y}$, the integral (A1) may be estimated as

$$
\mathrm{G}=\frac{\sigma_{y}(1+\cos \phi)}{(2 \pi)^{3 / 2} a_{1} a_{2}} \int_{-\infty}^{\infty} e^{-\left(u^{2} / 2 \sigma_{u}^{2}\right)} d u,
$$

where $\sigma_{u}=a_{2} /\left(\sigma_{y} a_{3}\right)$.

After integration, an explicit expression for the geometric factor at the optimal crabbing angle $\theta=\phi / 2$ reads

$$
\mathrm{G}=\frac{1}{2 \pi \sqrt{\sigma_{z}^{2}+\sigma_{z 0}^{\prime 2}} \sqrt{\sigma_{x}^{2} \sec ^{2} \frac{\phi}{2}+\sigma_{x 0}^{\prime 2}+\sigma_{y}^{\prime 2} \tan ^{2} \frac{\phi}{2}}} .
$$

If the laser pulse were ellipsoidal, the same result will be obtained. 
[1] S. Araki et al., Physics/0509016, KEK Report No. 200560, CLIC note 639, LAL 05-94.

[2] E. Bulyak et al., Phys. Rev. ST Accel. Beams 9, 094001 (2006).

[3] Super B Conceptual Design Report No. INFN/AE-07/02, SLAC-R-856, LAL 07-15.

[4] J. A. Aguilar-Saavedra et al. (ECFA/DESY LC Physics Working Group), arXiv:hep-ph/0106315; F. Richard, J. R. Schneider, D. Trines, and A. Wagner, arXiv:hep-ph/ 0106314.

[5] J. Daiton et al., in Proceedings of the 11th European Particle Accelerator Conference, Genoa, 2008 (EPS-AG, Genoa, Italy, 2008), p. 1903.

[6] A. Variola et al., in Proceedings of the 23rd Particle Accelerator Conference, Vancouver, Canada, 2009 (IEEE, Piscataway, NJ, 2009), MO6RFP068.

[7] B. Badelek et al., Int. J. Mod. Phys. A 19, 5097 (2004).

[8] G. Wormser et al., ELI-Nuclear Physics Workshop, Bucharest, 2010, http://www.eli-np.ro/documents/ eli-excom-meeting/Wormser-Gamma-ray-source-workinggroup.pdf.

[9] http://www.lynceantech.com/.

[10] ThomX Conceptual Design Report, LAL/RT 09-28, Soleil/SOU-RA-2678, edited by A. Variola, A. Loulergue, and F. Zomer.

[11] D. J. Gibson et al., Phys. Plasmas 11, 2857 (2004).

[12] M. Ando and C Uyama, Medical Application of Syncrotron Radiation (Springer-Verlag, Tokyo, 1998).

[13] F. Carroll, J. Cell. Biochem. 90, 502 (2003).

[14] E. G. Bessonov et al., arXiv:physics/0405003.

[15] D. E. Moncton, http://accelconf.web.cern.ch/Accelconf/ fls06/TALKS/PLT05_TALK.PDF.

[16] P. Walter et al., C.R. Physique 10, 676 (2009).

[17] M. Uesaka, http://www.physics.ucla.edu/PAHBEB2005/ talks/11_oct_2005/plenary_speakers/m_uesaka.pdf.

[18] C.P. J. Barthy and F. V. Hartemann, LLNL laboratory Report No. UCRL-TR 206825.

[19] F. Zimmermann et al., in Proceedings of the 11th European Particle Accelerator Conference, Genoa, 2008, Ref. [5], p. 2847.

[20] E. Bulyak and V. Skomorokhov, Phys. Rev. ST Accel. Beams 8, 030703 (2005).
[21] M. Xie, K-J. Kim, and A. Sessler, Nucl. Instrum. Methods Phys. Res., Sect. A 355, 163 (1995).

[22] A. Variola, in PosiPol Workshop 2006, CERN, Geneva 2006, http://indico.cern.ch/getFile.py/access? contribId=32\&session $I d=64 \&$ res $I d=1 \&$ material $I d=$ slides \& confId $=845$.

[23] E. Bulyak, in Proceedings of the 9th European Particle Accelerator Conference, Lucerne, 2004 (EPS-AG, Lucerne, 2004), WELPT138.

[24] P. Gladkikh, E. Bulyak, T. Omori, and J. Urakawa, in PosiPol 2009, Lyon, France (2009), http://indico.cern.ch/ getFile.py/access? contribId=19\&session $I d=11 \&$ resId $=$ $1 \&$ materialId $=$ slides \& confId $=53079$.

[25] J. Urakawa, Nucl. Instrum. Methods Phys. Res., Sect. A (2010)..

[26] H. Kogelnick and T. Li, Appl. Opt. 5, 1550 (1966).

[27] R. J. Jones and J. C. Diels, Phys. Rev. Lett. 86, 3288 (2001).

[28] R. J. Loewen, Ph.D. dissertation, Stanford University, Stanford, CA, 2003.

[29] G. Rempe et al., Opt. Lett. 17, 363 (1992).

[30] F. Zomer, Habilitation a Diriger de Recherches, LAL (2006).

[31] N. Falletto et al., Nucl. Instrum. Methods Phys. Res., Sect. A 459, 412 (2001).

[32] H. Shimizu, J. Phys. Soc. Jpn. 78, 074501 (2009).

[33] K. Sakaue, Ph.D. thesis, Wesada University, Japan, 2009.

[34] E. Roser et al., Opt. Lett. 30, 2754 (2005).

[35] A.E. Siegman, Lasers (University Science Books, Sausalito, California, 1986).

[36] F. Zomer et al., Appl. Opt. 48, 6651 (2009).

[37] Y. Honda et al., Opt. Commun. 282, 3108 (2009).

[38] J.A. Arnaud and H. Kogelnik, Appl. Opt. 8, 1687 (1969).

[39] S. J.M. Habraken, Phys. Rev. A 75, 033819 (2007).

[40] M. A. Furman, in Proceedings of PAC91, San Francisco, 1991, p. 422.

[41] M.A. Furman and M.S. Zisman, in Handbook of Accelerator Physics and Engineering, edited by A.W. Chao and M. Tigner (World Scientific, Singapore, 2006), Chap. 4.1. 